\title{
Instrumented flow-following sensor particles with magnetic position detection and buoyancy control
}

\author{
Sebastian Felix Reinecke ${ }^{1}$ and Uwe Hampel ${ }^{1,2}$ \\ ${ }^{1}$ Helmholtz-Zentrum Dresden-Rossendorf (HZDR), 01328 Dresden, Germany \\ ${ }^{2}$ AREVA Endowed Chair of Imaging Techniques in Energy and Process Engineering, Technical University of \\ Dresden, 01062 Dresden, Germany \\ Correspondence to: Sebastian Felix Reinecke (s.reinecke@hzdr.de)
}

Received: 19 February 2016 - Revised: 31 May 2016 - Accepted: 31 May 2016 - Published: 17 June 2016

\begin{abstract}
A concept for buoyancy control and magnetic position detection has been developed for the improvement of instrumented flow-following sensor particles. The sensor particles are used for investigation of hydrodynamic and biochemical processes in large-scale vessels such as biogas fermenters, bioreactors and aerated sludge basins. Neutral buoyancy of the sensor particles is required for tracing of the fluid flows. Buoyancy control is performed by adjustment of the sensor particles' volume, which is altered by an integrated piston. A miniaturized linear actuator, namely a stepper motor with linear transmission, is operated by a microcontroller to drive the piston. The buoyancy control unit enables accurate automated taring of the sensor particles in the stagnant process fluid to achieve neutral buoyancy. Therefore, the measured vertical position of the sensor particle as a function of the hydrostatic pressure is used as feedback. It has an incremental density change of $0.0136 \%$ as compared to water and a residual drift velocity of approximately $3.6 \times 10^{-3} \mathrm{~m} \mathrm{~s}^{-1}$. Furthermore, a minimum density of $926 \mathrm{~kg} \mathrm{~m}^{-3}$ can be set by full extension of the piston, which allows floating of the sensor particles after a defined event, namely critical charge of battery, full data storage or the end of a fixed time cycle. Thus, recovery of the sensor particles can proceed easily from the fluid level. The sensor particles with a buoyancy control unit are tested for depths up to $15 \mathrm{~m}$. Also, detection of a local magnetic position marker by the sensor particles has been implemented to enhance movement tracking. It was tested in a lab-scale biogas digester and was used for estimation of the liquid circulation time distribution and Peclét number to describe the macro-flow.
\end{abstract}

\section{Introduction}

Large-scale plants and vessels in the energy and process industry have limited accessibility for measurement instrumentation. Installation of local sensors with cabled connections is often not possible or avoided due to high costs. Furthermore, standard measurement techniques such as videometry, thermography, particle imaging velocimetry (PIV) and process tomography are not applicable to the process vessels due to the large vessel dimensions, the harsh environmental conditions and the opaque fluids. Thus, investigation, modelling and control of the chemical, mechanical and thermal processes are limited and often insufficient.

Temperature profiles, $\mathrm{pH}$ profiles, gas-liquid-solid distribution in the substrate and local digestion rate are of main interest for estimation of the process efficiency and for development and design of plants in the field of stirred bioreactors, fermenters and biological waste water treatment facilities. Moreover, hydrodynamic parameters - e.g. flow velocity, distribution of dead zones and short-circuit flows, and circulation times - are in focus for characterization of processes.

Application of instrumented flow followers and autonomous sensor technologies allows investigation of the aeration, mixing and heating regimes by acquisition of a few basic physical parameters, such as temperature and pressure. This information is currently only available at a few local measurement positions. Thus, design and operation of plants and processes are mainly based on the experience of the operator. Therefore, autonomous sensor technologies are get- 
ting more relevant for the process industry (Antoniou et al., 2009).

Autonomous sensor particles were developed by Thiele et al. (2010) to be applied to large-scale vessels - such as bioreactors, biogas digesters and activated sludge basins - as instrumented flow followers that continuously capture the spatially distributed process parameters and provide the data to an external computer system for further analysis. The sensor particles have been tested under realistic flow conditions of stirred tanks and biogas digesters, and characteristic process parameters have been estimated, e.g. vertical flow velocity, circulation time, circulation number, Peclét number and degree of suspension (Reinecke et al., 2012; Reinecke, 2014).

The configuration of the sensor particles by Thiele et al. (2010) comprises a set of sensors for basic parameters, namely immersions depth as a function of ambient pressure, 3-D acceleration and temperature. Therefore movement tracking of the sensor particles was limited to the vertical position and vertical velocity. An important feature of flowfollowing sensor particles is sufficient tracing of the fluid flows. Therefore, neutral buoyancy of the sensor particles is required to avoid flow offsets from the sensor particles' gravity and buoyancy. In practice, neutral buoyancy of particles for mixing process applications is related to a residual terminal velocity of the particles below $1 \times 10^{-2} \mathrm{~m} \mathrm{~s}^{-1}$ measured in stagnant fluids. The sensor particles' mass used to be adjusted manually to tare the sensor particles for a low sinking or rising velocity. Moreover, recovery was cumbersome due to the undefined final position of the sensor particles.

This paper presents an enhanced concept of sensor particles with an integrated buoyancy control unit and detection of external magnetic position markers. An autonomous taring regime is implemented in the sensor particles to achieve neutral buoyancy in a sample volume of the process substrate. Moreover, an event-driven floating of the sensor particles is possible with the buoyancy control unit, which allows recovery from the liquid surface.

Movement-tracking capabilities of the sensor particles are also extended to the horizontal direction by detection of external magnetic position markers. Transmitting coils are immersed into to vessel at a fixed position and excited by a modulated signal. The transmitted signal is captured by the sensor particles during passage at the marker position.

The enhanced sensor particles were tested under real flow conditions in a lab-scale biogas digester with an oval shape where the position marker was used to deliver information about the horizontal flow in the vessel.

\section{Sensor particle design}

The enhanced sensor particles are based on the original concept by Thiele et al. (2010) and comprise a robust housing with an integrated electronic measuring and control unit (Fig. 1). The integrated electronics include miniaturized sen-

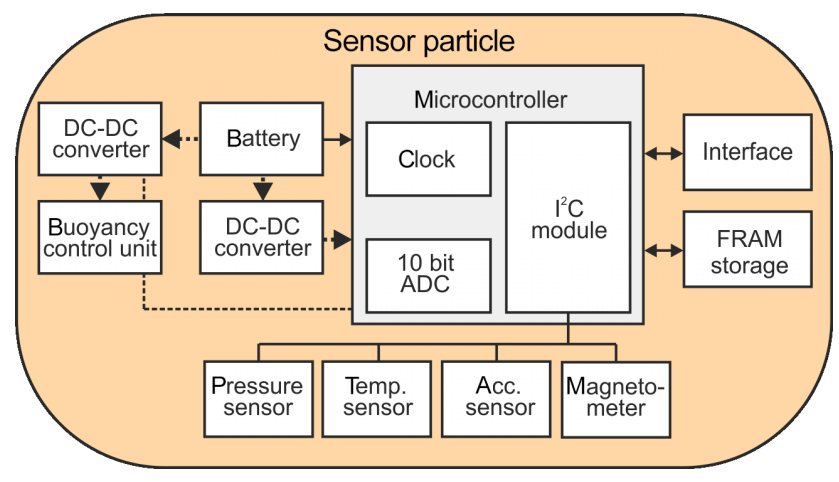

Figure 1. Components of a sensor particle with internal electronics and buoyancy control unit.

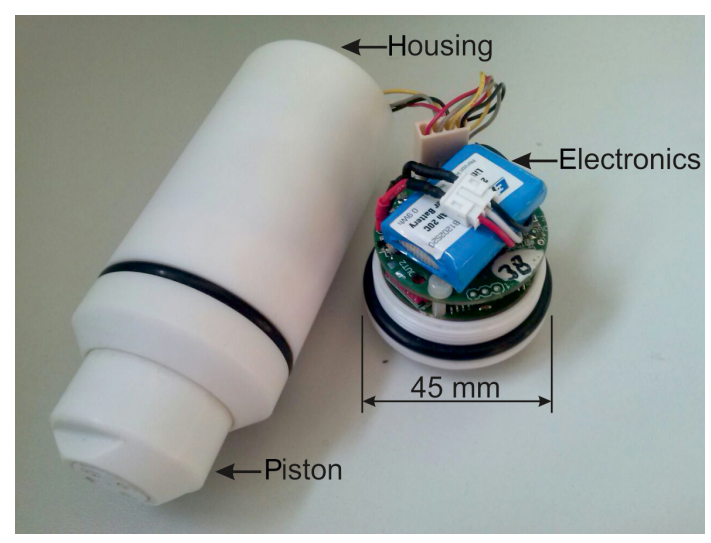

Figure 2. Open sensor particle with the electronics and the extended piston of the buoyancy control unit.

sors for temperature $\left(0 \ldots 50^{\circ} \mathrm{C}\right)$, vertical position as a function of hydrostatic pressure $(0 \ldots 300 \mathrm{kPa}), 3-\mathrm{D}$ acceleration $(-6 \ldots+6 \mathrm{~g})$ and magnetic field $(-4 \ldots+4 \mathrm{G})$, and a microcontroller that performs the autonomous taring and measuring regimes. A lithium polymer battery pack is connected to a voltage converter and works as an onboard energy supply.

The robust housing is modular to carry the electronics and the components of the buoyancy control unit. Figure 2 depicts the sensor particle housing with the extended piston and the connected electronics. The mounted sensor particles have a minimal volume of $V_{\mathrm{SP}}=1.5 \times 10^{-4} \mathrm{~m}^{-3}$, a mass of $m_{\mathrm{SP}}=0.145 \mathrm{~kg}$ and thus a density of $\rho_{\mathrm{SP}}=967 \mathrm{~kg} \times \mathrm{m}^{-3}$ without internal payload and retracted piston. The equivalent spherical diameter is $d_{\mathrm{SP}}=6.6 \times 10^{-2} \mathrm{~m}$, and the sphericity is $\psi=0.9$. Internally, a void of $1.7 \times 10^{-5} \mathrm{~m}^{3}$ is reserved for the payload which is set to $0.005 \mathrm{~kg}$ to achieve almost neutral buoyancy with a minimally extended piston in water. A maximal density of the sensor particles of $1873 \mathrm{~kg} \times \mathrm{m}^{-3}$ is achievable when the whole void is filled with the payload made of stainless steel (density $8000 \mathrm{~kg} \times \mathrm{m}^{-3}$ ). 


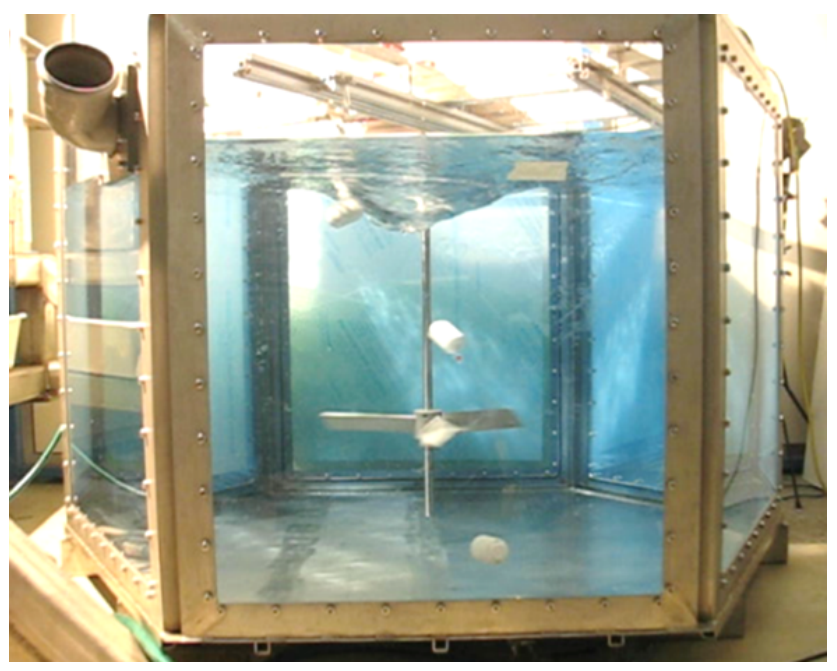

Figure 3. Characterization of the required density offset of sensor particles in a stirred vessel experiment in water for an improved recovery at the liquid surface.

\subsection{Buoyancy control concept}

The purpose of the buoyancy control unit is (i) to enable automated taring of sensor particles to achieve neutral buoyancy before the start of the autonomous measurement regime and (ii) to ensure an event-driven floating of sensor particles for recovery from the liquid surface. Recovery of sensor particles from the liquid surface is only possible if the sensor particles have sufficient buoyancy offset to float and remain at the liquid surface. A buoyancy offset of sensor particles implies a reduction of the particles' density. In industrial applications the fluids are in motion due to mixing by impellers or gas dispersion, and thus turbulent flows are present also at the liquid surface. This turbulence at the surface support resuspension of the sensor particles into the liquid body, and a higher buoyancy offset is necessary to keep the sensor particles floating. An experimental study was conducted under highly turbulent flow conditions in a stirred tank to estimate the required density offset of sensor particles to achieve sufficient buoyancy offset. Figure 3 depicts the stirred tank that was filled with water of density $\rho_{\mathrm{f}}=998 \mathrm{~kg} \mathrm{~m}^{-3}$ up to a filling level $\mathrm{H}_{0}=0.8 \mathrm{~m}$ and the suspended sensor particles. Strong axial forces that pull down the sensor particles are present at the intake region of the pitched-blade impeller in the circulation flow. Therefore, different impeller speeds $n$ were used, namely 0.92 , 1.23 and $1.53 \mathrm{~s}^{-1}$. These correspond to Reynolds numbers of $2.3 \times 10^{5}, 3.1 \times 10^{5}$ and $3.8 \times 10^{5}$, respectively, which meet the criterion $\operatorname{Re}>0.2 \times 10^{5} \times 5 \times 10^{5}$ for a fully turbulent flow in the stirred tank (Liepe et al., 1998; Schubert, 2003). Three sensor particles with the configuration by Thiele et al. (2010) were used with different taring, namely neutrally buoyant $\left(\rho_{\mathrm{SP}} \approx \rho_{\mathrm{f}}\right)$, offset of $2 \%\left(\rho_{\mathrm{SP}} \approx 0.98 \times \rho_{\mathrm{f}}\right)$ and offset of $6 \%\left(\rho_{\mathrm{SP}} \approx 0.94 \times \rho_{\mathrm{f}}\right)$.
Vertical residence profiles were extracted from the measured data according to Reinecke et al. (2010) to describe the residence of the sensor particles over the height of the liquid column (Fig. 4). The neutrally buoyant sensor particle $\left(\rho_{\mathrm{SP}} \approx \rho_{\mathrm{f}}\right)$ reflects the relevant residence profile to characterize the hydrodynamics of the process. An upwards shift of the profiles is recognized for the sensor particle with the offset of $2 \%\left(\rho_{\mathrm{SP}} \approx 0.98 \times \rho_{\mathrm{f}}\right)$. However, the residence at the liquid surface is still too low for improved recovery. A significant peak of the residence profile at the surface is achieved with the density offset of $6 \%\left(\rho_{\mathrm{SP}} \approx 0.94 \times \rho_{\mathrm{f}}\right)$, and only temporary resuspension was observed. In conclusion a density offset of $6 \%$ compared to the neutrally buoyant setting is required to ensure predominant floating of sensor particles even under highly turbulent flow conditions.

Based on the results of the experimental study, a buoyancy control concept for sensor particles has been developed. Figure 5 shows the main components. The housing has an integrated piston to adjust the volume and therefore also the buoyancy of the sensor particle. A miniaturized linear actuator, namely a stepper motor with integrated linear transmission and incremental step length of $0.0254 \mathrm{~mm}$, drives the piston and is powered by the onboard battery pack. The motor driver is directly controlled by the microcontroller. A feedback of the current vertical position of the sensor particle is provided by the pressure sensor which is required for automated taring. Floating of the sensor particle is set by the microcontroller after a defined event, namely critical charge of battery, full data storage or the end of a fixed time cycle.

The piston is integrated in the upper front of the sensor particle and is supported by an O-ring seal. The fitting of the seal was adjusted to reduce the force for actuation of the piston to below $10 \mathrm{~N}$ but still ensures tightness of the housing for water columns of up to $15 \mathrm{~m}$ (test pressure of $250 \mathrm{kPa}$; safety factor: 1.5). The linear transmission of the actuator is situated inside the hollow part of the piston and is connected to the upper end of it to use the full travel of the actuator of $15 \mathrm{~mm}$ for the piston. In this way, a volume change of $8 \%$ is possible as compared to the normal volume of the sensor particle, which results in a minimum density of $926 \mathrm{~kg} \mathrm{~m}^{-3}$, and floating of the sensor particles is ensured at full travel of the piston even under turbulent flow conditions in most aqueous fluids.

Neutral buoyancy can only be achieved if the incremental density change is sufficiently small. Due to the small step length of the linear actuator, the incremental density change of the buoyancy unit is $0.0136 \%$. An estimate of the resulting maximal particle terminal velocity can be calculated by Eq. (1):

$v_{\mathrm{T}}=v_{*}\left(\frac{\rho_{\mathrm{f}}^{2}}{\mathrm{~g} \times \mu_{f} \times\left(\rho_{\mathrm{SP}}-\rho_{\mathrm{f}}\right)}\right)^{-\frac{1}{3}}$

with the liquid viscosity represented by $\mu_{f}$, the dimensionless terminal velocity by $v_{*}=\mathrm{f}\left(d_{*}, \psi\right)$ and the dimen- 
(a)

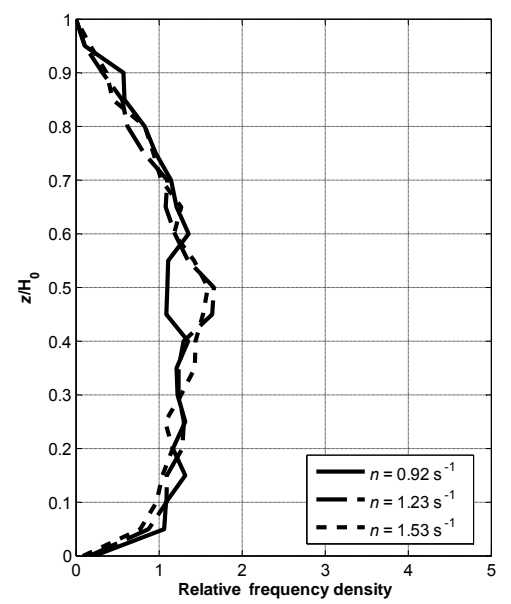

(b)

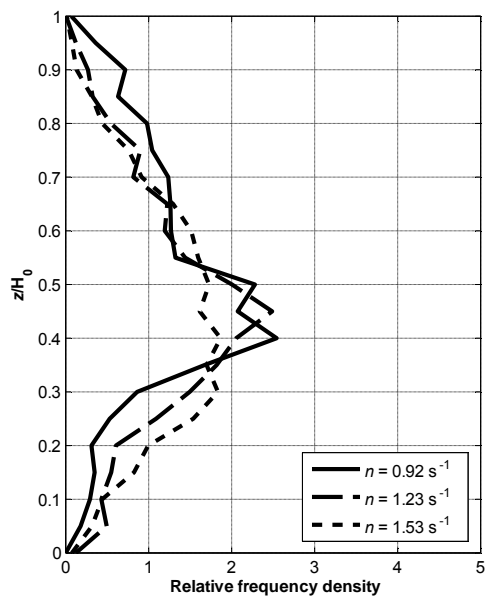

(c)

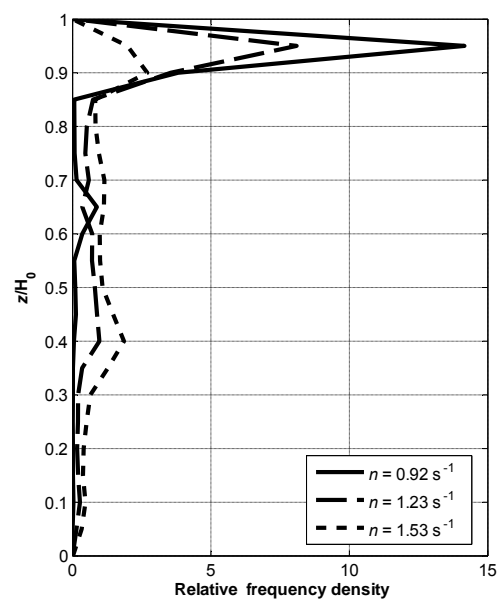

Figure 4. Vertical residence profiles of the sensor particles from the experiments in the stirred vessel for characterization of the required density offset at the impeller speeds of $0.92,1.23$ and $1.53 \mathrm{~s}^{-1}$ with different density $\rho_{\mathrm{SP}}$ of the sensor particles at the fluid density $\rho_{\mathrm{f}}$ : (a) $\rho_{\mathrm{SP}} \approx \rho_{\mathrm{f}}$, (b) $\rho_{\mathrm{SP}} \approx 0.98 \times \rho_{\mathrm{f}}$ and (c) $\rho_{\mathrm{SP}} \approx 0.94 \times \rho_{\mathrm{f}}$.

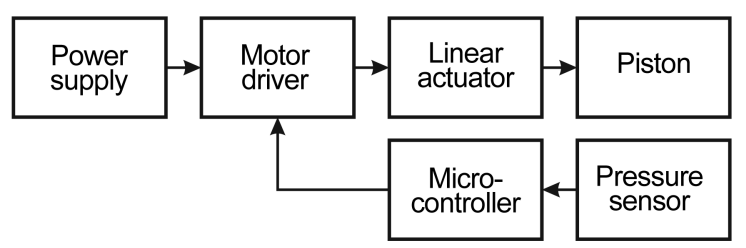

Figure 5. Components of the buoyancy control unit of sensor particles.

sionless diameter by $d_{*}$ according to Haider and Levenspiel (1989). Assuming $\mu_{f}=1 \times 10^{-3} \mathrm{~Pa} \times \mathrm{s}$ for water and $\mu_{f}=1 \mathrm{~Pa} \times \mathrm{s}$ as effective viscosity for typical liquid biosubstrates, the expected maximal terminal velocity of sensor particles is $1 \times 10^{-2} \mathrm{~m} \mathrm{~s}^{-1}$ and $3 \times 10^{-4} \mathrm{~m} \mathrm{~s}^{-1}$, respectively. This is below the acceptable limit of $1 \times 10^{-2} \mathrm{~m} \mathrm{~s}^{-1}$ for neutral buoyancy in mixing processes and shows that the incremental density change of the buoyancy unit is sufficient for taring of sensor particles. An automated taring regime of sensor particles has been implemented in the microcontroller that uses the signal of the pressure sensor as feedback for the current vertical position. This regime proceeds prior to the application of sensor particles to the process vessel. At first each sensor particle is inserted into a mobile container, i.e. a column of ca. $1 \mathrm{~m}$ height, which is filled with a sample volume of process substrate, and the sensor particles detect the immersion from the pressure signal (Fig. 6a). The piston of the buoyancy control unit is initially retracted. That makes the sensor particle sink slightly, which is recognized by the sensor particle. The sensor particle remains at the bottom of the container for a short duration for thermal adjustment of the housing to the substrate temperature. Then the piston is extended stepwise, and the hydrostatic pressure is measured after a duration of $250 \mathrm{~ms}$, which is the empirically estimated relaxation time $t_{\min }$ of the sensor particle for incremental extension of the piston in water. For $t_{\min }<200 \mathrm{~ms}$ in water the density change is faster than the sensor particle body responds, and thus the maximal amplitudes of the vertical sensor particle movement starts to reach the container height during taring due to the low liquid viscosity. In the period of $t_{\min }$ the incremental density change of $0.0136 \%$ gives a change of the vertical position of $3 \times 10^{-3}$ and $8 \times 10^{-5} \mathrm{~m}$ with regard to the estimated maximal terminal velocity of sensor particles in water and in biosubstrate, respectively.

The piston is further extended until the sensor particle slowly starts to rise and the current pressure value gets lower than the maximum pressure at the container bottom (Fig. 6b). Then comes the control regime where the position of the piston is adjusted with regard to $t_{\min }$ until the pressure difference of consecutive measurements remains zero; i.e. it constantly falls below the resolution of the pressure sensor of $9 \mathrm{~Pa}$ and $9 \times 10^{-3} \mathrm{~m}$ in vertical position. Thus, the sensor particle is neutrally buoyant with a minimal tolerance after the automated taring regime. The resolution of $9 \times 10^{-3} \mathrm{~m}$ and the relaxation time $t_{\min }$ result in a residual terminal velocity of approximately $3.6 \times 10^{-3} \mathrm{~m} \mathrm{~s}^{-1}$, which is a hypothetical estimate. Also this value is below the limit of $1 \times 10^{-2} \mathrm{~m} \mathrm{~s}^{-1}$ for neutral buoyancy in mixing processes. However, only a negligible terminal velocity of the sensor particles has been observed during application of the automated taring regime under laboratory conditions. Achievement of such results is cumbersome with manual buoyancy adjustment of sensor particles and takes a lot of effort. 


\subsection{Magnetic position detection}

Enhanced movement tracking of sensor particles includes detection of a local position marker that is located at a fixed position in the process vessel, by an onboard sensor. Bryant (1969) and Day (1975) applied a similar concept for estimation of fluid circulation times in bioreactors for the first time. They used a flow-following radio pill, and the emitted radio signal was detected inside the vessel by a locally installed antenna. Position marking for sensor particles is based on an immersible coil that is mounted into the cross section of the flow in the process vessel to extend the movementtracking abilities of the sensor particles. The coil is made of a cable spool which is mounted in a watertight pipe frame. It has a width of $0.53 \mathrm{~m}$ and a height of $0.78 \mathrm{~m}$. This simple construction allows scaling of the coil from laboratory up to industrial applications. It emits a magnetic signal in a limited range, and this signal is detected by a magnetic sensor of the sensor particle during passage of the coil. A magnetometer is used in the current configuration to measure the ambient magnetic flux generated by the coil. Nevertheless, the influence of the magnetic background - namely earth's magnetic field, metallic vessel components and other surrounding magnetic sources - is also measured. Thus, either sufficiently high magnetic signal levels above the magnetic background level or signal modulation/coding are required for confident position detection at the coil. A simple frequency hop excitation signal is used at the coil to achieve a clear discrimination of the position marker from the magnetic background. The measured magnetic flux signal $B$ at the magnetometer in the sensor particle is correlated with the reference signal $B_{\text {ref }}$ according to Eq. (2):

$\hat{R}_{\mathrm{XY}}(m)=\sum_{n=0}^{N-m-1} B(n+m) B_{\mathrm{ref}}^{*}(n)$,

where $n$ and $m$ are the discrete time steps and $\mathrm{N}$ the length of

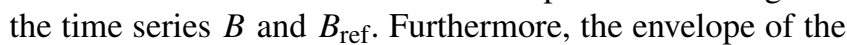
correlated sequence $\hat{R}_{\mathrm{XY}}(\mathrm{m})$ is extracted to apply a robust threshold binarization of the signal.

The signal frequency changes from 1 to $2 \mathrm{~Hz}$ in a period of $2 \mathrm{~s}$. A low-frequency band was chosen to ensure correct acquisition of the marker signal also at lower acquisition rates of the autonomous measurement regime. However, due to the small bandwidth of the excitation signal the signal correlation may be too low in certain flow situations. Higher processing gain of the signal detection may be achieved with more advanced modulation techniques that require a higher signal bandwidth, such as spread spectrum techniques in digital modulation. However, this in turn requires higher computational performance and energy consumption of the electronics, which reduces the runtime of sensor particles.

Therefore the position marker was designed to generate a higher magnetic flux than the magnitude of earth's magnetic field of $0.5 \mathrm{G}$ even in the centre of the coil. In combination (a)

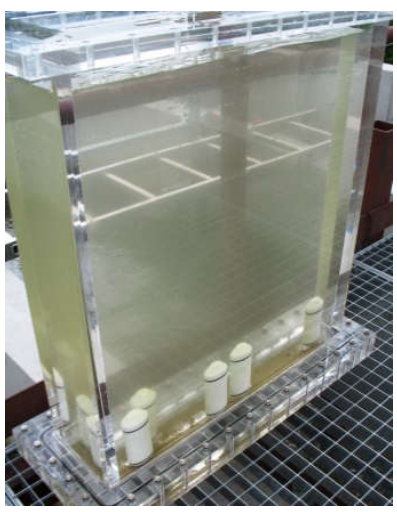

(b)

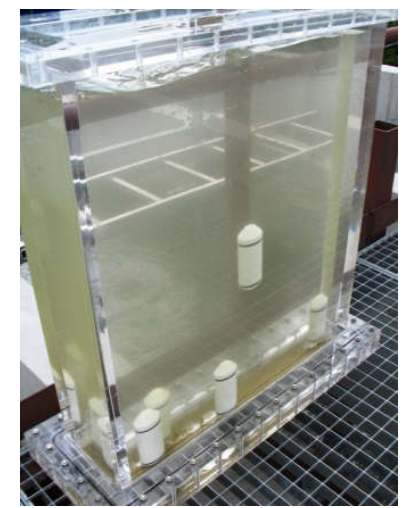

Figure 6. Sensor particles during the automated taring regime in a fluid sample: (a) residence at the container bottom and (b) elevated position during the automated taring regime.

with the simple signal modulation this allows clear detection of the passage of the sensor particles at the coil. Furthermore, the magnetic position detection of sensor particles extends the abilities of sensor particles to estimate individual circulation times also in horizontal flow situations. That is a characteristic process parameter for mixing in agitated tanks.

\section{Experiment in a lab-scale biogas digester}

\subsection{Experimental set-up}

Advanced sensor particles with a buoyancy control unit and magnetic position marker were tested in a lab-scale biogas digester. The digester has an oval geometry and is operated as a circulation reactor to achieve homogeneous and mild mixing of the substrates in the biogas digester (Fig. 7). Two impellers are installed horizontally at the long sides of the digester in the centre of the channel. The filling volume is $2 \mathrm{~m}^{3}$, and the channel width is $0.55 \mathrm{~m}$. Both impellers were driven at the impeller tip speed of 5.5 and $6.7 \mathrm{~m} \mathrm{~s}^{-1}$. An aqueous xanthan solution with a concentration of $5 \mathrm{~g} \mathrm{~L}^{-1}$ was used to simulate the rheology of real biogas substrates. The coil was installed at the apex of the vessel (Fig. 7b). Four sensor particles performed the automated taring regime and were then applied to the digester at an acquisition rate of $8 \mathrm{~s}^{-1}$. They remained in the process for $100 \mathrm{~min}$ before final floating and recovery from the liquid surface. Afterwards the measured digital values were downloaded from the data storage of the sensor particles for further analysis on an external computer.

\subsection{Floating and recovery of sensor particles}

After the programmed measurement period of $100 \mathrm{~min}$, full extension of the piston was initiated by the buoyancy control unit on all sensor particles for final floating. The first sensor particles already remained at the liquid surface before 
(a)

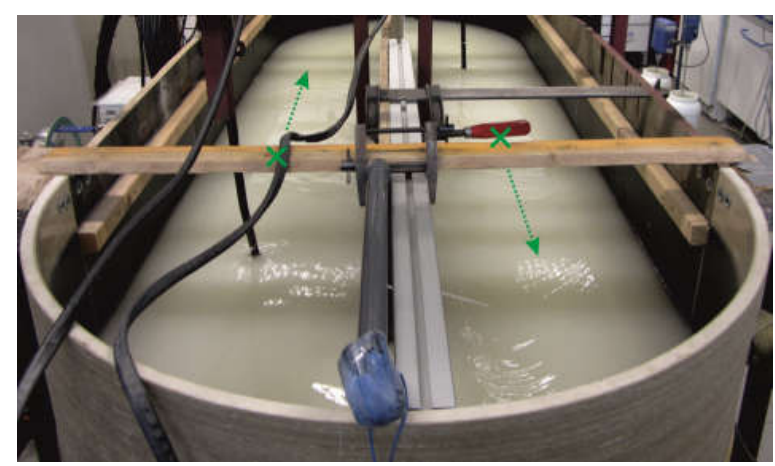

(b)

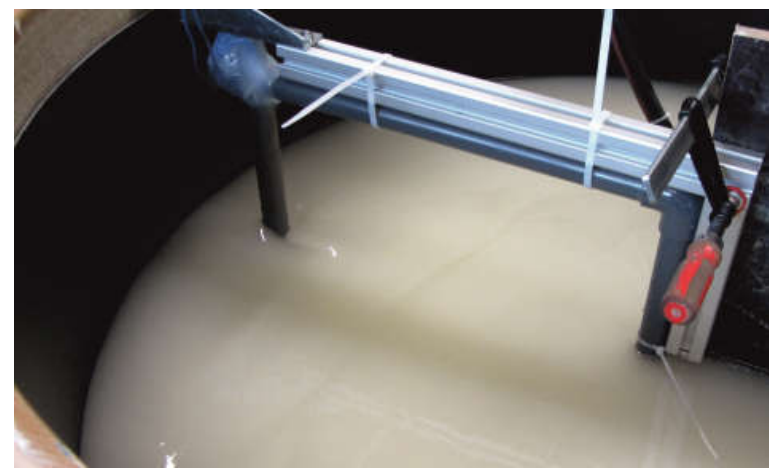

Figure 7. Lab-scale biogas digester with oval geometry and an installed coil as a position marker. The horizontal positions of the two impellers and the main flow direction are marked in (a).

the piston was fully extended when they entered a stagnant zone (see Fig. 8a). After full extension of the piston, the sensor particles were clearly visible at the liquid surface (see Fig. 8b) and were recovered manually by means of a dip net. No resuspension of sensor particles was observed with a fully extended piston even in turbulent flow regions (see Fig. 8c)

\subsection{Detection of position marker}

The time series of the measured magnetic signal contains the received signal of the position marker and is influenced by the movement of the sensor particles in earth's magnetic field. Figure 9a depicts a sequence of the measured magnetic signal of a sensor particle in the biogas digester. Passages at the position marker can already be seen from the higher signal magnitudes above $0.5 \mathrm{G}$. A clear discrimination of the passages is achieved by cross-correlation of the measured magnetic signal with the reference signal according to Eq. (2), which can be seen from the extracted envelope of the correlated signal sequence in Fig. 9b. Binarization of the correlated signal provides further reduction of the received signal (Fig. 9c). The threshold was adjusted to avoid false detection of sensor particle movement in earth's magnetic field. Therefore, a separate test measurement was performed in the (a)

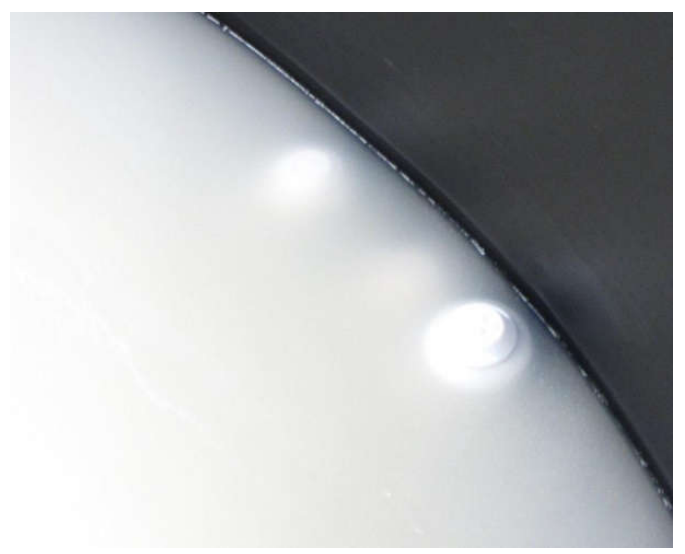

(b)

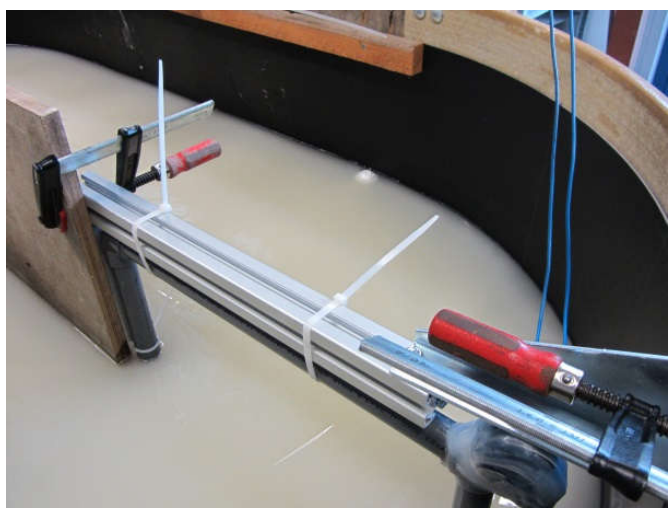

(c)

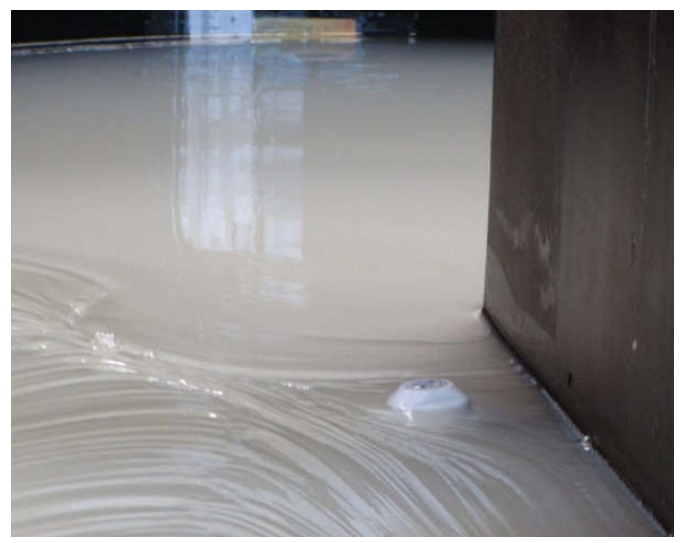

Figure 8. Floating sensor particles at different positions on the liquid surface in the biogas digester.

digester without position marker, and the threshold value was minimized. Finally, the temporal differences in the binary signal represent the horizontal circulation times of the sensor particles in the digester. 


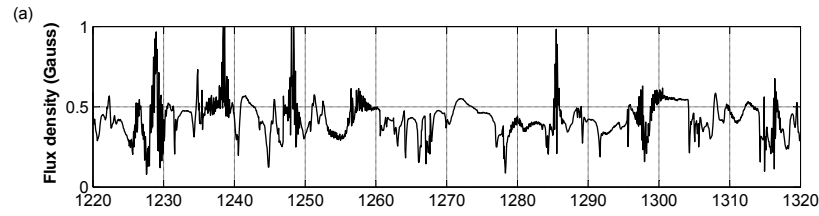

(b)

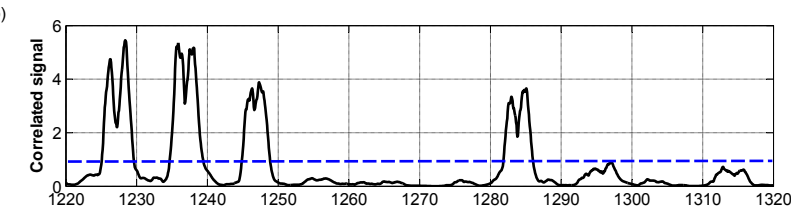

(c)

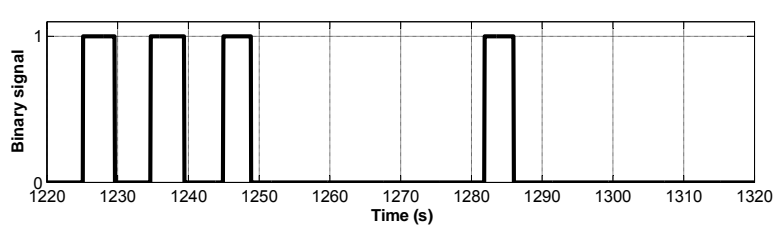

Figure 9. Sequence of the analysed magnetic field signal of a sensor particle for detection of a position marker for the horizontal flow in the biogas digester: (a) magnitude of the magnetic flux density, (b) correlated signal with the threshold for binarization and (c) binary signal.

\subsection{Estimation of circulation time distribution and dispersion}

The population of the individual circulation times $t_{\mathrm{c}}$ yields the circulation time distribution which is depicted in Fig. 10 for both impeller speeds. All four sensor particles show a similar shape of the circulation time distribution which supports the reliability of the data. An even more significant result is obtained by the analysis of the combined time series data of all four sensor particles which is also depicted in Fig. 10.

The influence of the impeller speed can be observed simply from the shape of the circulation time distributions. A rather flat distribution is obtained for the lower impeller speed, where the average value is $\bar{t}_{\mathrm{c}}=56.0 \mathrm{~s}$ and the standard deviation is $\sigma_{\mathrm{c}}=66.1 \mathrm{~s}$. In contrast, the distribution is more distinct for the higher impeller speed with $\bar{t}_{\mathrm{c}}=25.7 \mathrm{~s}$ and $\sigma_{\mathrm{c}}=23.5 \mathrm{~s}$.

According to Luo and Al-Dahhan (2008) these statistical quantities of the circulation time distribution estimate the Peclét number:

$P e \approx 2 \cdot\left(\frac{\bar{t}_{\mathrm{c}}}{\sigma_{\mathrm{c}}}\right)^{2}$,

which is the ratio of the advective flow to diffusion and thus describes back mixing of mass transfer. An ideal stirred tank reactor has $P e=0$ (ideal mixing), and an ideal plug flow reactor has $P e=\infty$ (convection and no back mixing). The values of the circulation time distribution yield $P e=1.4$ for the lower impeller speed and $P e=2.4$ for the higher impeller speed. This indicates a slight reduction of back mixing and (a)

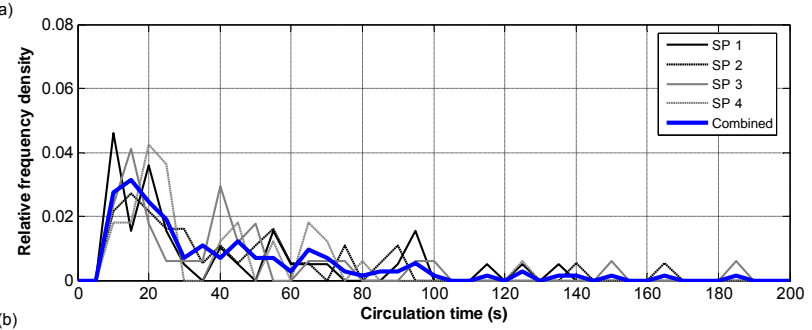

(b)

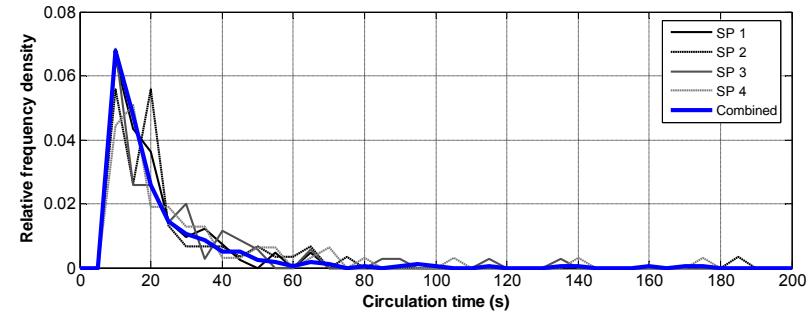

Figure 10. Circulation time distribution of all four sensor particles (SP1 ...4) in the biogas digester at the impeller speeds (a) 5.5 and (b) $6.7 \mathrm{~m} \mathrm{~s}^{-1}$. The distribution of the combined time series of all sensor particles is given in blue.

an increased convective flow after the increase of the impeller speed. In conclusion, the extracted values from the sensor particle measurements - namely the average circulation time, the standard deviation and the Peclét number - describe the macro-flow conditions in the biogas digester and indicate that the sole increase of impeller speed in the biogas digester experiment did not contribute to an improved mixing in this vessel geometry.

\section{Conclusions}

Enhanced instrumented flow-following sensor particles have been developed with an integrated buoyancy control unit and magnetic position detection. The buoyancy control unit enables automated taring by a sensor particle to achieve neutral buoyancy in the process fluid without cumbersome manual adjustments of payloads. Furthermore, the sensor particles are capable of floating to the liquid surface after a certain event, such as critical charge of battery, full data storage or the end of a fixed time cycle, for easy recovery. This facilitates the handling during application of sensor particles to processes in laboratory set-ups and especially in vessels of real plants in the energy and process industry.

The movement-tracking ability of sensor particles is extended by detection of a magnetic position marker, namely an immersible coil. The marker is placed at a fixed position in the process vessel to cover horizontal movement of sensor particles in addition to the vertical position as a function of the ambient pressure.

Four sensor particles with a buoyancy control unit and magnetic position detection were successfully tested in a labscale biogas digester with horizontally circulation flow. The 
measured data describe the macro-flow in the digester. Magnetic position detection of sensor particles was used to estimate the individual circulation times and the circulation time distribution in the horizontal flow of the digester. Moreover, back mixing is characterized by estimation of the Peclét number from the circulation time statistics of sensor particles.

Performance of the magnetic position detection can be improved by implementation of advanced signal modulation techniques, such as spread spectrum techniques. However, this requires thorough design of the data acquisition unit in the sensor particles. Furthermore, it will be investigated whether the position marker concept can be combined with an inertial measurement unit in the sensor particles to achieve a position-tracking system with 3-D capabilities. However, signal coding for detection of multiple position markers distributed across the vessel will be considered as well. Nevertheless, accuracy of the estimated position has to be investigated for development of a reliable position-tracking system.

Acknowledgements. Results of this paper also originate from the research project LEOBEL, which is funded by the German Federal Environmental Foundation (DBU - Deutsche Bundesstiftung Umwelt) under the reference number AZ30799. The experiment in the lab-scale biogas digester was conducted in cooperation with the Fraunhofer Institute for Ceramic Technologies and Systems Dresden (IKTS).

Edited by: S. Zimmermann

Reviewed by: two anonymous referees

\section{References}

Antoniou, M., Boon, M. C., Green, P. N., Green, P. R., and York, T. A.: Wireless Sensor Networks for Industrial Processes, Proceedings of IEEE SAS, New Orleans, LA, USA, 13-18, 2009.

Bryant, J.: Mixing in Fermenters, Dissertation, University of Cambridge, Camdridge, UK, 1969.

Day, A.: Mixing in stirred tanks, Dissertation, University of Exeter, Exeter, UK, 1975.

Haider, A. and Levenspiel, O.: Drag coefficient and terminal velocity of spherical and nonspherical particles, Powder Technol., 58, 63-70, 1989.

Liepe, F., Sperling, R., and Jembere, S.: Rührwerke, Theoretische Grundlagen, Auslegung und Bewertung, 1. Aufl., Eigenverlag Fachhochschule Köthen, Köthen, Germany, 1998.

Luo, H.-P. and Al-Dahhan, M. H.: Macro-mixing in a draft-tube airlift bioreactor, Chem. Eng. Sci., 63, 1572-1585, 2008.

Reinecke, S., Deutschmann, A., Jobst, K., Kryk, H., Friedrich, E., and Hampel, U.: Flow following sensor particles - Validation and macro-mixing analysis in a stirred fermentation vessel with a highly viscous substrate, Biochem. Eng. J., 69, 159-171, doi:10.1016/j.bej.2012.09.010, 2012.

Reinecke, S. F.: Instrumentierte Strömungsfolger zur Prozessdiagnose in gerührten Fermentern, Dissertation, Dresdner Beiträge zur Sensorik, Band 52, TUDpress, Dresden, Germany, 2014.

Schubert, H.: Handbuch der Mechanischen Verfahrenstechnik, Wiley-VCH, Verlag, Weinheim, Germany, 2003.

Thiele, S., Da Silva, M. J., and Hampel, U.: Autonomous sensor particle for parameter tracking in large vessels, Meas. Sci. Technol., 21, 085201 1-8, doi:10.1088/0957-0233/21/8/085201, 2010. 